\title{
Preparation of micro-encapsulated strawberry fragrance and its application in the aromatic wallpaper
}

\author{
Zuobing Xiao, Yu Zhang, Guangyong Zhu*, Yunwei Niu, Ziqi Xu, Jiancai Zhu \\ Shanghai Institute of Technology, No. 100 Haiquan Road, 201418, Shanghai, China \\ "Corresponding author: e-mail: gyz@sit.edu.cn
}

\begin{abstract}
Micro-encapsulated strawberry fragrance was successfully prepared with wall materials including maltodextrin, sodium octenylsuccinate and gum Arabic. The micro-capsule was added to wallpaper and aromatic wallpaper with strawberry characteristics was obtained. The particle distribution, surface morphology, chemical structure, thermal property and controlled release performance of micro-encapsulated fragrance and aromatic wallpaper were investigated using laser particle size analyzer, scanning electron microscopy (SEM), Fourier transform infrared spectroscopy (FT-TR), thermal gravity analysis (TGA) and chromatography-mass spectrometer (GC-MS). The results showed that the average diameter of micro-capsule was $2 \mu \mathrm{m}$ and the particles mainly distributed in the tissues of wallpaper. The result of TGA showed that the micro-capsule had a good stability. Meanwhile, the aromatic wallpaper had strawberry aroma more than 3 months and took on excellent controlled release performance.
\end{abstract}

Keywords: micro-encapsulation; fragrance; wallpaper; controlled release.

\section{INTRODUCTION}

Since micro-encapsulation technology occurred in the middle of 20th century, more and more researchers devoted their time into the field of micro-encapsulation. As an outcome of cross-disciplinary such as physics, chemistry and material, micro-encapsulation technology has permeated majority areas of peoples' daily life ${ }^{\mathbf{1 - 4}}$. Broadly speaking, micro-encapsulation technology is a production method of micro-particle using one or more wall materials to encapsulate core materials via physical, chemical or physicochemical ways. By this way, core materials were protected from rapidly volatilizing, degradation or oxidization by light, air or other factors. Microencapsulation process results in a solid or liquid particle with the diameter ranging from several microns to hundreds of microns ${ }^{5-7}$. Ordinarily, the phase state of core materials can be gas, liquid or solid. The wall materials generally were classified natural macromolecular and the synthetic macromolecule. In general, natural macromolecular have the advantages of safety, nontoxicity, degradation and biocompatibility compared with synthetic macromolecule. The most commonly used natural macromolecules are chitosan, starch, dextrin and alginate ${ }^{8-13}$. Sodium octenylsuccinate is also called purity gum belonging to starch. It is a polymer composed of hydrophilic polysaccharide long chain and hydrophobic octenyl side chain. Its space stability mechanism makes itself a fine emulsifier ${ }^{14}$. Furthermore, sodium octenylsuccinate has the advantages of low-cost and reproducibility. Gum Arabic pertains to natural macromolecule amylose and broadly used as emulsion stabilizer. It has strong tolerance toward temperature and $\mathrm{pH}^{\mathbf{1 5}}$. Acting as a starch hydrolysis product, maltodextrin is also widely used in micro-encapsulation ${ }^{\mathbf{1 6}}$.

With the development of peoples' life level, odor time is coming quietly. From food to daily chemical products, flavored products have drawn extensive attention and highly praise. Fragrance plays an important role in the national economy and the people's livelihood. However, fragrance usually has poor heat stability and is volatile, easily oxidized. This limits its application. Luckily, the occurrence of micro-encapsulated technology provides a good idea for solving this problem. So far, a variety of micro-capsules has been produced and used in life. $\mathrm{Hu}$ et al. ${ }^{17}$ prepared micro-encapsulated Jasmine fragrance using melamine resin as the wall material and added it in textile. Their research illustrated that the aromatic textile could keep aroma for half a year and its aroma holding time was six times as that textile handed with normal fragrance. Sánchez-Navarro et al. ${ }^{\mathbf{1 8}}$ produced fragrant leather using melamine formaldehyde resin as wall material via in-situ polymerization and assessed its rubbing performance.

In this paper, maltodextrin, sodium octenylsuccinate and gum Arabic were selected as wall materials to encapsulate strawberry fragrance. A high-pressured homogenizer was used in the encapsulation process to highly disperse wall materials, overcome the surface tension and increase the contact area between water phase and oil phase ${ }^{19}$. All the materials we used in this article are carbohydrates. They are healthy, non-poisonous, environmentally friendly, low cost and easy to be obtained ${ }^{\mathbf{2 0}}$. Moreover, the whole process is simple to operate and has good repeatability. The products were added to indoor wallpaper to prepare a new one with the strawberry aroma. The aroma wall paper can improve the indoor air environment, boost air quality, and expand market of wallpaper industry. Therefore, the additional value of wallpaper was increased. So far, the study about the aromatic paper by means of micro-encapsulated technology has rarely been reported. Yi et al. ${ }^{\mathbf{2 1}}$ prepared sandalwood rice paper and explored its controlled release performance with the electronic nose. But their observation time was only 10 days. In this study, the aroma content analysis and controlled release have been investigated for 90 days. In addition, micro-capsules were characterized by laser particle size analyzer, scanning electron microscopy, Fourier transform infrared spectroscopy and thermal gravity analysis. All these measurements offered some basic data for promotion and application of aromatic wallpaper in the market. 


\section{EXPERIMENTS}

\section{Materials}

Maltodextrin (DE degree: 10-15) was purchased from Dongxiao Biological Technology Co. Ltd (Shandong zhucheng, China). Purity gum 2000 came from National Starch Co. Ltd (Shanghai, China). Gum Arabic (AR) was obtained from Aladdin Biological Technology Co. Ltd (Shanghai, China). Styrene-butadiene latex and wallpaper were provided by Qifeng Specialty Paper Co. Ltd (Shandong, China). Strawberry fragrance and deionized water were prepared by our lab.

\section{Preparation of strawberry micro-encapsulated fragrance}

Firstly, $35 \mathrm{~g}$ maltodextrin was dissolved in $34 \mathrm{~g}$ deionized water at $60^{\circ} \mathrm{C}$ and then stirred at a speed of $600 \mathrm{rpm} /$ min for $20 \mathrm{~min}$ to form a solution. Secondly, $7 \mathrm{~g}$ purity gum and $3 \mathrm{~g}$ gum Arabic were added into the above solution and stirred at a speed of $600 \mathrm{rpm} / \mathrm{min}$ for 40 min to form the wall material solution. After cooling to room temperature, $20 \mathrm{~g}$ strawberry fragrance was added into the wall material solution, stirring at a speed of $600 \mathrm{rpm} / \mathrm{min}$ for $20 \mathrm{~min}$. Then, the mixture was sheared using high-pressured homogenizer at a speed rate of $10000 \mathrm{rpm} / \mathrm{min}$ for $20 \mathrm{~min}$ to obtain micro-encapsulated strawberry fragrance. By freezing drying in $-38^{\circ} \mathrm{C}$ for $48 \mathrm{~h}$, dried samples were obtained for further FTIR and thermogravimetric analysis.

\section{Application of micro-encapsulated strawberry fragrance in wallpaper}

Styrene-butadiene latex and deionized water were mixed in a proportion of 3:1. A micro-encapsulated strawberry fragrance which mass was $1 \%$ of styrene-butadiene latex was added to the above mixture and stirred for $30 \mathrm{~min}$ to completely distribute the micro-encapsulated fragrance into the latex. Thus, the homogeneous coating liquid was obtained. The K Control Coater (see Fig. 1) was used to add coating liquid to wallpaper. At first, blank wallpaper was formed on the pallet. Secondly, the red rubber head winding bar was installed allowing coating thickness $12 \mu \mathrm{m}$. Thirdly, $30 \mathrm{ml}$ coating liquid was poured onto the wallpaper. The wallpaper was fully saturated with the coating liquid for $1 \mathrm{~min}$. After that, the button was switched on to make the bar proof for wallpaper at a constant rate and pressure. Through the above steps, the aromatic wallpaper with uniform micro-encapsulated fragrance coating thickness was obtained. The wet fragrant wallpaper was dried in the air at room temperature. The preparation of wallpaper with the normal fragrance followed the same method as above.

\section{Morphology and chemical structure characterization}

The strawberry micro-encapsulated fragrance was diluted with deionized water to a concentration $10 \%$. Then, the diluent was poured into the sample cell of Zetasizer Nano Laser Particle Size Analyzer (Marvin, Britain) to measure the diameter and size distribution of micro-capsule.

The blank wallpaper and micro-encapsulated fragrant wallpaper were adhered respectively to the conducting resin. Before the observation, the specimens were coated with $\mathrm{Au}$ and its acceleration voltage was $10 \mathrm{KV}$. After the

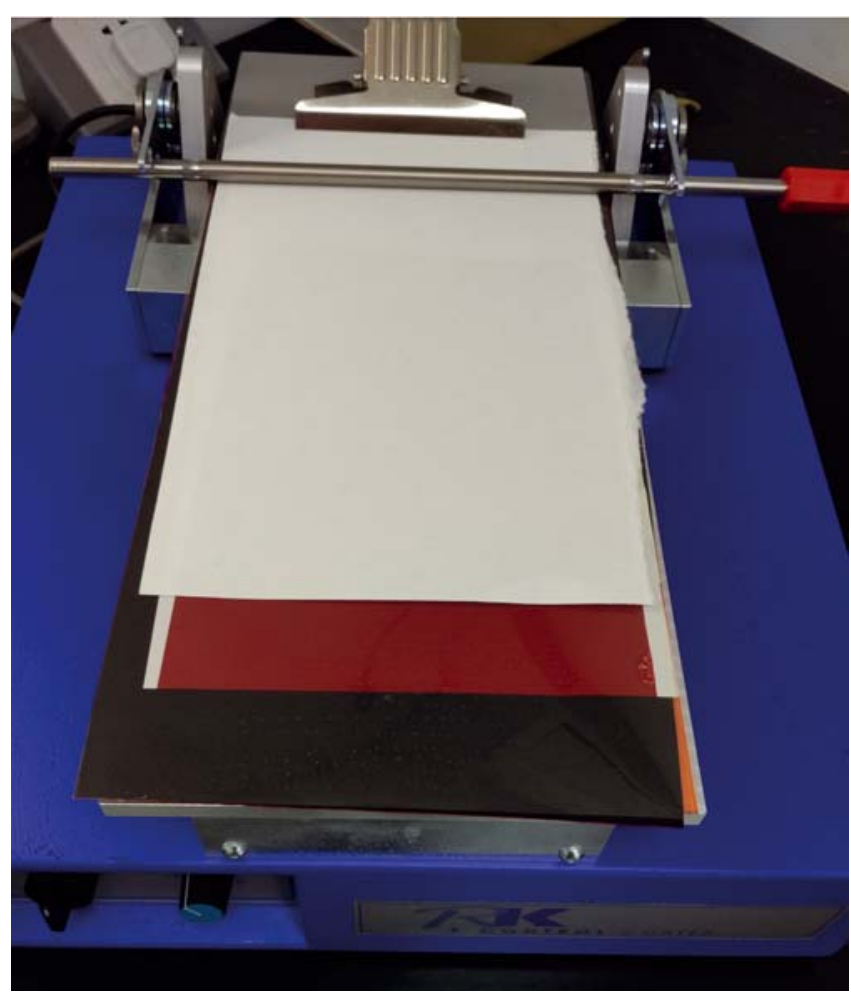

Figure 1. Photo of coater applied to the preparation of aromatic wallpaper

pretreatment, the Phenom Desktop Scanning Electron Microscope (FEI, America) was used to observe the morphology of blank wallpaper and micro-encapsulated fragrant wallpaper.

The strawberry fragrance, micro-encapsulated strawberry fragrance, blank wallpaper and the micro-encapsulated aromatic wallpaper were scanned at full-wave band by means of Fourier infrared spectrometer (Bruke, Germany) in the range of $500-4000 \mathrm{~cm}^{-1}$. The obtained infrared spectrograms were used to investigate the chemical structures and their structural changes caused by the interactions.

The thermogravimetric analyzer (Q5000IR, TA, America) was used to investigate the heat stability of strawberry fragrance; freezing dried micro-encapsulated fragrance and blank micro-encapsulated fragrance. The whole process was carried out with the protection of $\mathrm{N}_{2}$. Temperature programming was controlled ranging from room temperature to $600^{\circ} \mathrm{C}$ at the heating rate of $10^{\circ} \mathrm{C} / \mathrm{min}$.

\section{Controlled release investigation about wallpaper}

Gas chromatograph - mass spectrometer (7890A/5975C, Agilent) was used to investigate the fragrance release of wallpaper. $1.0 \mathrm{~g}$ micro-encapsulated aromatic wallpaper and aromatic wallpaper (treated with normal fragrance) were put in $15 \mathrm{~mL}$ headspace vials, respectively. These vials were kept at room temperature for 1 day, 3 days, 7 days, 15 days, 25 days, 40 days, 60 days and 90 days respectively. 5 uL 2-methoxy-4-methyl phenol was added into the vials as the internal standard. The aged extraction fiber was inserted in the vials to absorb the fragrance released from the treated wallpaper. The length of extraction fiber exposed to the head-space vial was $1.6 \mathrm{~cm}$. And the extraction time was $120 \mathrm{~min}$. The extraction fiber was quickly inserted into the injection 
port of GC-MS and the time of sample injection sustained for $5 \mathrm{~min}$.

The conditions of head-space solid phase-micro-extraction were as follows: the aging temperature of extraction was $250^{\circ} \mathrm{C}$. The extraction fiber was aged for $20 \mathrm{~min}$ in $\mathrm{N}_{2}$ flow at the rate of $1.0 \mathrm{~mL} / \mathrm{min}$.

The condition of gas chromatography-mass spectrometry was as follows: the chromatographic column was DB-Innowax capillary column $(60 \mathrm{~m} \times 0.25 \mathrm{~mm} \times 0.25$ $\mu \mathrm{m}$, Agilent). Helium (purity $=99.999 \%$ ) was selected as carrier gas at a rate of $2.0 \mathrm{~mL} / \mathrm{min}$. Column oven adopted temperature programming. Firstly, it started at $40^{\circ} \mathrm{C}$ maintaining for $6 \mathrm{~min}$. Secondly, the emperature rose at the rate of $3^{\circ} \mathrm{C} / \mathrm{min}$ to $100^{\circ} \mathrm{C}$. And then, the temperature rose at the rate of $5^{\circ} \mathrm{C} / \mathrm{min}$ to $230^{\circ} \mathrm{C}$ keeping for $10 \mathrm{~min}$. Electron bombardment ion source was used. The electron energy was $70 \mathrm{eV}$. The spectra collection mode was full scanning at the range of 30-450 amu. The solvent delayed for $3.00 \mathrm{~min}$.

The qualitative and quantitative determination method of chemical compound was as follows: The mass spectrums of unknown compounds were compared with the spectrum libraries of NIST and WILEY 7n (Agilent Technologies Inc.). The content of each compound was calculated by area normalization method of peak area.

\section{RESULTS AND DISCUSSION}

\section{Particle size distribution and morphology analysis}

The micro-capsule liquid prepared above was put into the sample cell for particle size distribution analysis. It was repeated three times. Figure 2 represented the results. As for the whole particle size distribution shape, a normal distribution can be observed. Particle size distribution ranged from $1 \mu \mathrm{m}$ to $2.5 \mu \mathrm{m}$. And the mean diameter of micro-capsule was 2 um. A narrow unimodal distribution demonstrated that the system was stable and homogeneous ${ }^{22}$. Figure 3 was the scanning electron microscope photos of blank wallpaper and micro-encapsulated aromatic wallpaper at the same magnification times. The micro-structure of wallpaper can be seen from Figure 3. A series of disorderly wood

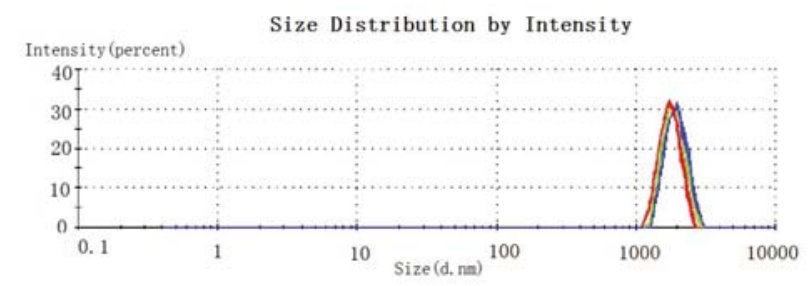

Figure 2. Particle size distribution of micro-encapsulated fragrance

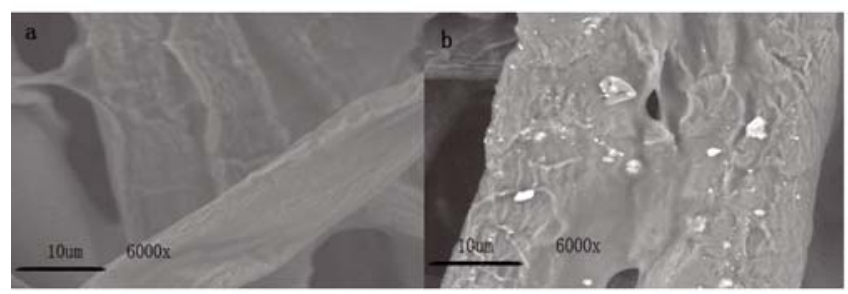

Figure 3. SEM images of the blank wallpaper (a) and microencapsulated aromatic wallpaper (b) fibers constituted the wallpaper. After the aromatic treatment, micro-capsules were glued at the wood fibers of wallpaper. The size of particles stuck at the wood fibers was in accord with the result of particle size distribution analysis. All these results demonstrated the successful preparation of micro-capsules and aromatic wallpaper.

\section{FT-IR ANALYSIS}

Figure 4 shows the infrared spectrograms of micro-encapsulated strawberry fragrance, blank wallpaper, micro-encapsulated strawberry aromatic wallpaper and strawberry fragrance. Compared with strawberry fragrance and micro-encapsulated strawberry fragrance, 5 characteristic absorption peaks had relatively large changes (namely: the stretching vibration absorption peak at $3396 \mathrm{~cm}^{-1}$ of $\mathrm{O}-\mathrm{H}$ bond, the stretching vibration at $2925 \mathrm{~cm}^{-1}$ of $\mathrm{C}-\mathrm{H}$ bond in the group of $-\mathrm{CH}_{2}{ }^{23}$, the stretching vibration at $1737 \mathrm{~cm}^{-1}$ of $\mathrm{C}=\mathrm{O}$ bond in the carboxylic acid or esters, the stretching vibration at $1226 \mathrm{~cm}^{-1}$ of $\mathrm{C}-\mathrm{O}$ bond in esters and the stretching vibration at $1031 \mathrm{~cm}^{-1}$ of $\mathrm{C}-\mathrm{X}$ bond). From the spectrograms of micro-encapsulated fragrance and normal fragrance in Figure 4, the changes in the absorption strength were observed. The absorption strength of strawberry micro-encapsulated fragrance was stronger than that of the normal strawberry fragrance. Owing to the coating action of wall material towards the core material, the infrared absorption of strawberry fragrance was obstructed. Therefore, weak absorption strength of micro-encapsulated strawberry fragrance was observed. Furthermore, other changes such as the disappearing, bath chromic shift and hypo chromatic shift were also observed. For example, the absorption peak at $1737 \mathrm{~cm}^{-1}$ of $\mathrm{C}=\mathrm{O}$ bond in aldehydes materials disappeared; the absorption peak at $2925 \mathrm{~cm}^{-1}$ moved into $2936 \mathrm{~cm}^{-1}$. All of these changes were caused by the interaction between the wood fibers and the micro-encapsulated fragrance. Hence, these results indirectly testified the strawberry fragrance was successfully embedded in the cavity of micro-capsule. The difference was not obvious between the infrared spectrograms of blank wallpaper and micro-encapsulated aromatic wallpaper as Figure 4. The fragrance has a strong odor in spite of low dose. In consideration of the trait of fragrance, only $1 \%$ of

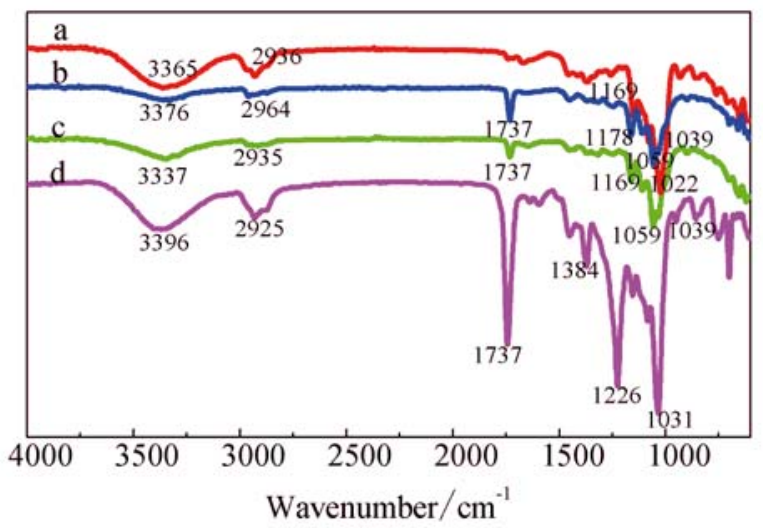

Figure 4. FTIR spectra of microencapsulated fragrance (a), blank wallpaper (b), microencapsulated aromatic wallpaper (c) and fragrance (d) 
micro-encapsulated fragrance was added into the wallpaper. So, the biggest difference between the wallpaper and micro-encapsulated aromatic wallpaper was odor rather than the chemical structure. Therefore, the consequence of an unambiguous distinction between blank wallpaper and micro-encapsulated aromatic wallpaper was observed. The wallpaper was macromolecular polysaccharide cellulose and its structural unit was glucose. Therefore, some of the absorption peaks of wallpaper existed in the range of $1200 \mathrm{~cm}^{-1}$ to $950 \mathrm{~cm}^{-124}$, such as absorption peaks at $1178 \mathrm{~cm}^{-1}, 1059 \mathrm{~cm}^{-1}$ and 1039 $\mathrm{cm}^{-1}$. For aroma wallpaper, the absorption peaks at $3376 \mathrm{~cm}^{-1}, 2964 \mathrm{~cm}^{-1}$ and $1178 \mathrm{~cm}^{-1}$ were all moved to the direction of the long wave. It was the direction of characteristic absorption peaks of micro-encapsulated fragrance. These appearances manifested that micro-encapsulated fragrance was absorbed in wallpaper. The strength of the absorption peak at $3376 \mathrm{~cm}^{-1}$ was because of the overlay of absorption peak of micro-encapsulated fragrance. The absorption peaks of stretching vibration at $1737 \mathrm{~cm}^{-1}$ in wallpaper, stretching vibration at 1059 $\mathrm{cm}^{-1}$ of $\mathrm{C}-\mathrm{X}$ bond in wallpaper, stretching vibration at $1039 \mathrm{~cm}^{-1}$ of C-O-C of the pyranoid ring of wallpaper were not changed in position and strength ${ }^{25}$. This indicated that the chemical structure of wallpaper was not changed. And the aromatic treatment of wallpaper was worked relying on the hydrogen bonds or the van der Waals forces of intermolecular physical interaction.

\section{TG analysis}

Figure 5 shows the thermogravimetric curves of strawberry fragrance, strawberry micro-encapsulated fragrance and blank micro-encapsulated wall material respectively. From Figure 5a, it can be seen that the mass of strawberry fragrance was gradually lost with the increase of temperature and the majority of mass lost at the scope of $60-140^{\circ} \mathrm{C}$. The maximum thermal decomposition speed happened at $112.9^{\circ} \mathrm{C}$. When the temperature rose to $200^{\circ} \mathrm{C}$, strawberry fragrance was volatilized completely and there was no remnant. From Figure $5 \mathrm{~b}$ and Figure $5 \mathrm{c}$, three steps of thermal decomposition for strawberry micro-encapsulated fragrance and blank micro-capsule can be observed. First of all, it took place below $250^{\circ} \mathrm{C}$. In this phase, strawberry micro-encapsulated fragrance lost $6.35 \%$ while the blank micro-capsule lost $8.23 \%$ in mass. The thermal decomposition of this step was

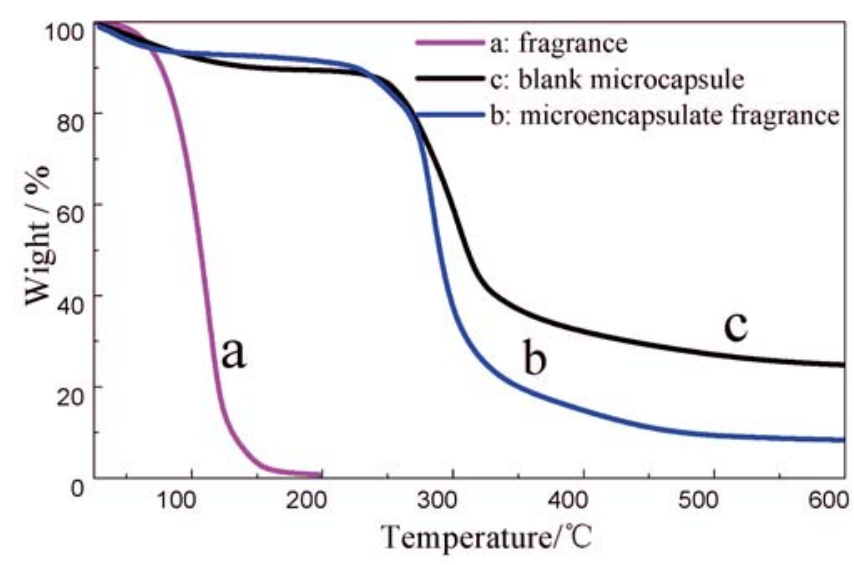

Figure 5. TG curves of fragrance (a), microencapsulated fragrance (b) and blank microcapsule (c) occurred due to the lost of free water and fragrance absorbing at the surface of micro-capsules. Secondly, the thermal decomposition was at the range of $240-600^{\circ} \mathrm{C}$. Two stages consisted of this phase. The formal stage was corresponding to the rapid release of the core material with the maximum decomposition rate at $282.5^{\circ} \mathrm{C}$. The maximum decomposition rate appeared at $307.84^{\circ} \mathrm{C}$ of the latter stage which caused by the decomposition of wall material. In consequence of embedding of the wall material, the maximum volatile rate temperature of core material changed from $112.9^{\circ} \mathrm{C}$ to $282.5^{\circ} \mathrm{C}$. However, with the temperature increase, the core material in the cavity brought about the increase of vapor pressure. The vapor pressure made the gap existing in the surface of micro-capsule bigger than before, and thus, the core material quickly leaked from the micro-capsule cavity ${ }^{26}$. Compared with the thermal decomposition curves of strawberry fragrance and micro-encapsulated strawberry fragrance, we knew that the micro-encapsulated strawberry fragrance possessed good thermal stability. This guaranteed the stability of the micro-encapsulated strawberry fragrance in the application of aromatic wallpaper.

\section{GC-MS analysis of the controlled release of aromatic wallpaper}

Controlled release of micro-encapsulated fragrance is an important parameter to confirm the effect of micro-encapsulation. The micro-encapsulated strawberry fragrance was added in wallpaper. The obtained aromatic wallpaper was expected to have controlled and sustained release pleasure odor. Figure 6 shows the results of aroma content changes with the increase of storage time for two type aromatic wallpaper. From the Figure 6, we knew that the aroma content of aromatic wallpaper appeared continuous reduction from 0 to 90 days. The fragrance is easily volatile. After the homogeneous coating at the wallpaper via physical interaction, the strawberry fragrance was rapidly volatilized with the bigger contact area between the fragrance and air. Therefore, in the initial phase, the aroma content of the aromatic wallpaper was bigger than the micro-encapsulated aromatic wallpaper' in spite of at the same fragrance adding amount. However, with the increase of time, the amount of residual fragrance in the aromatic wallpaper was less and less. At 40 days, no fragrance

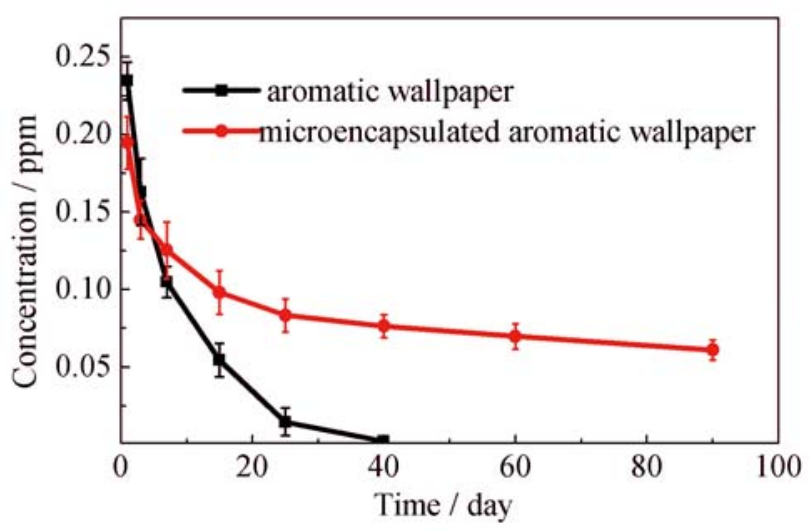

Figure 6. Fragrance release curves of aromatic wallpaper and microencapsulated aromatic wallpaper 
in the aromatic wallpaper can be detected by GC-MS. When operating GC-MS analysis, sensory evaluation was conducted at the same time. From the Figure 6, we learned that the aroma content of the micro-encapsulated aromatic wallpaper represented relatively a fast release rate in the first three days. Afterwards, the aroma content loss rate was gradual decline. The former fast release rate was caused by the fragrance which was not totally embedded into the cavity of micro-capsule or glued at the surface of the particle. This fragrance rapidly released on the condition of no protective layer like micro-encapsulated wall material. The later stage corresponds to the process of controlled release of micro-encapsulated fragrance. Owing to the protection of wall material of micro-capsule, the volatility of fragrance reduced greatly. The micro-encapsulated aromatic wallpaper was still kept fragrant at 90 days. Figure 6 illustrated that the micro-encapsulated fragrance had good controlled release performance. And the fragrance holding time of micro-encapsulated aromatic wallpaper could reach three months. This finding was in accord with the result of Liu et al. ${ }^{27}$. All these experiment results indicated that the micro-encapsulated fragrance can be used in the production of aroma wallpaper.

\section{CONCLUSION}

In summary, a micro-encapsulated strawberry fragrance was prepared using the maltodextrin, purity gum 2000 and gum Arabic as wall material. This micro-encapsulated strawberry fragrance was used in wallpaper and aroma wallpaper was obtained. This aroma wallpaper has the characteristics of improving the air quality, increasing the additional value and enlarging the market of wallpaper. The mean diameter of the micro-capsule was $2 \mu \mathrm{m}$. The Fourier infrared spectrometer showed that there was just molecular interaction rather chemical reaction between the micro-encapsulated fragrance and wallpaper. After the aromatic treatment, the chemical structure of wallpaper was not changed. The contrast of thermogravimetric curves of strawberry fragrance, strawberry micro-encapsulated fragrance and blank micro-encapsulated wall material confirmed the successful preparation and good stability of micro-capsule. The controlled release performance detection of the aromatic wallpaper and the micro-encapsulated aromatic wallpaper via GC-MS illustrated that the micro-encapsulated aromatic wallpaper possessing an excellent ability of controlled release performance. The fragrance holding time of the micro-encapsulated aromatic wallpaper could maintain three months.

\section{ACKNOWLEDGMENTS}

This work was financially supported by the National Key R\&D Program (2016YFA0200300), Shanghai Plateau Discipline "Chemical Engineering and Technology (Perfume and Aroma Technology) and the National Natural Science Fund of China (No. 21276157, No. 21476140).

\section{LITERTURE CITED}

1. Xiao, Z., Liu, W., Zhu, G., Zhou, R. \& Niu, Y. (2014). A review of the preparation and application of flavour and essential oils microcapsules based on complex coacervation technology. J. Sci. Food Agric. 94, 1482-1494. DOI: 10.1002/ jsfa.6491.

2. Behzadnasab, M., Esfandeh, M., Mirabedini, S.M., Zohuriaan-Mehr, M.J. \& Farnood, R.R. (2014). Preparation and characterization of linseed oil-filled urea-formaldehyde microcapsules and their effect on mechanical properties of an epoxy-based coating. Colloid. Surf. $A$ 457, 16-26. DOI: 10.1016/j.colsurfa.2014.05.033.

3. Mirabedini, S.M., Dutil, I. \& Farnood, R.R. (2012). Preparation and characterization of ethyl cellulose-based core-shell microcapsules containing plant oils. Colloid. Surf. A 394, 74-84. DOI: 10.1016/j.colsurfa.2011.11.028.

4. Xiao, Z., Wang, E., Zhu, G., Zhou, R. \& Niu, Y. (2016). Preparation, characterization and rheological behavior of chitosan nanocapsule emulsion encapsulated tuberose fragrance. Pol. J. Chem. Technol. 18, 1-8. DOI: 10.1515/pjct-2016-0021.

5. Carvalho, I.T., Estevinho, B.N. \& Santos, L. (2016). Application of microencapsulated essential oils in cosmetic and personal healthcare products-a review. Int. J. Cosmetic Sci. 38, 109-119. DOI: 10.1111/ics.12232.

6. Nelson, G. (2001). Microencapsulation in textile finishing. Rev. Prog. Color. 31, 57-64. DOI: 10.1111/j.1478-4408.2001. tb00138.x.

7. Han, L.L., Hou, Z.Q., Wen, J., Wu, Y.M., Qian, Y.Y. \& Gong, S.L. (2016). Preparation and evaluation of high-loaded perilla seed oil by microencapsulation with different wall materials. Sci. Tech. Food Ind. 37(8), 167-170. DOI: 10.13386/j. issn1002-0306.2016.08.026

8. Chen, H.M., Wei, O.Y., Lawuyi, B.S., Martoni, C. \& Prakash, S. (2005). Reaction of chitosan with genipin and its fluorogenic attributes for potential microcapsule membrane characterization, J. Biomed. Mater. Res. 75, 917-927. DOI: 10.1002/jbm.a.30492.

9. Syah, I.T., Darmadji, P. \& Pranoto, Y. (2015). Microencapsulation of refined liquid smoke using maltodextrin produced from broken rice starch. J. Food Process. Pre. 40(3), 437-446. DOI: 10.1111 jfpp.12621.

10. Ziar, H., Gérard, P. \& Riazi, A. (2012). Calcium alginate-resistant starch mixed gel improved the survival of Bifidobacterium animalis subsp. lactis Bb12 and Lactobacillus rhamnosus LBRE-LSAS in yogurt and simulated gastrointestinal conditions. Int. J. Food Sci. Tech. 47, 1421-1429. DOI: 10.1111/j.1365-2621.2012.02989.x.

11. Kim, S., Jo, A. \& Ahn, J. (2010). Application of chitosan-alginate microspheres for the sustained release of bacteriophage in simulated gastrointestinal conditions. Int. J. Food Sci. Tech. 50, 913-918. DOI: 10.1111/ijfs.12736.

12. Ma, Y., Zhang, Y., Zhao, S., Wang, Y., Wang, S., Zhou, Y., Li, N, Xie, H., Yu, W., Liu, Y., Wang, W. \& Ma, X. (2012). Modeling and optimization of membrane preparation conditions of the alginate-based microcapsules with response surface methodology. J. Biomed. Mater. Res. 100A, 989-998. DOI: $10.1002 / \mathrm{jbm} . \mathrm{a} .34032$.

13. Lee, B.B., Ravindra, P. \& Chan, E.S. (2013). Size and shape of calcium alginate beads produced by extrusion dripping. Chem. Eng. Technol. 36, 1627-1642. DOI: 10.1002/ ceat. 201300230 .

14. Zhao, H.M., Chen, X. \& Wu, J. (2001). The preparation, properties and using of starch sodium alkenylsuccinate. Cereal Food Ind. 1, 23-27. DOI: 10.3969/j.issn.1672-5026.2002.01.008.

15. Hu, L. (2010). Study on the mciroencapsulation of fish oil using Chitosan/ Gum acacia complex coacervation method. Master dissertation, Ocean University of China, Shangdong, China.

16. Ge, X., Fei, X. Q., Chen, Y., Luo, F. \& Wang, Y.P. (2013). Properties of oil-tea camellia seed oil microcapsule with different wall materials. China Oil. Fat. 38, 26-29. DOI: 10.3969/j.issn.1003-7969.2013.02.007. 
17. Wang, L. (2008). Study on aroma microcapsule effect in textile finishing. Master dissertation, Qiangdao University, Shandong, China.

18. Sánchez-Navarro, M.M., Pérez-Limiñana, M.Á., Arán-Ais, F. \& Orgilés-Barceló, C. (2015). Scent properties by natural fragrance microencapsulation for footwear applications. Polym. Int. 64, 1448-1464. DOI: 10.1002/pi.4941.

19. Dong, H. \& Chen, B. (2008). Studies on effects of high-pressure homogenization on stability of microencapsulated lipid lotion. Beverage Ind. 11(7), 17-21. DOI: 10.3969/j. issn.1007-7871.2008.07.006.

20. Zhang, J., Zhang, W., Wei, F., Zhang, F. \& Wang, S. (2012). Preparation and characterization of conjugated linolec acids encapsulated into compound encapsulant materials. $J$. Chinese Oil. Assoc. 27(1), 70-74. DOI: 10.3969/j.issn.10030174.2012.01.016.

21. Chen, Y. (2013). Preparation of sandalwood microcapsule and its application to aroma xuan paper. Master dissertation, Shanghai Institute of Technology, Shanghai, China.

22. Lu, J. (2013). Study on stability and rheological properties of oil in water emulsions prepared by food grade pure gum. Master dissertation, Zhejiang Gongshang University, Zhejinag, China.

23. Liu, Z., Tan, Z. \& Huang, H. (2016). Preparation and characterization of cellulose hydrogels from tea shoots of different maturity levels. Mod. Food Sci. Tech. 32(4), 166-170. DOI: $10.13982 /$ j.mfst.1673-9078.2016.4.027.

24. Wang, M. (2013). Study on qualitative and quantitative method of blended fiber in infrared spectrum, Master dissertation, Zhejiang Sci-Tech University, Zhejiang, China.

25. Sui, X., Li, C., Xia, X., Zhou, R., Wang, Y. \& Feng, Y. (2016). Photocatalytic degradation property of hemicellulose/TiO2 composite gel. CIESC J. 67(5), 2070-2077. DOI: 10.11949/j.issn.0438-1157.20151542.

26. Liu, C., Chen, K., Ji, L. \& Zhu, J. (2015). Preparation of epoxy resin coated ammonium polyphosphate microcapsules and their flame retardant effects on polypropylene. Acta Mat. Composit. Sin. 32(3), 728-736. DOI: 10.13801/j.cnki. fhclxb.201503.007.

27. Miao, H. (2012). Flavor microcapsules: Preparation and application in decorative paper. Master dissertation, Tian University of Science and Technology, Tianjin China. 